significant results of OC treatment. The main tasks of OC epidemiology are: continuation of in-depth studies of the prevalence in the regions of the Republic of Kazakhstan with the identification of population groups and regions with the lowest and highest rates of morbidity and mortality from OC. Disclosures Epidemiological data on the incidence rates of malignant neoplasms of the ovaries according to Globocan 2018 show significant differences across countries (per 100,000 women): from 3.8 in Central Africa to 11.9 in Central and Eastern Europe [1].

\section{NEOADJUVANT CHEMOTHERAPY OR FIRST LINE DEBULKING SURGERY IN ADVANCED OVARIAN CANCERS}

${ }^{1}$ Chemseddine Chekman, ${ }^{2}$ Fatiha Gouaref, ${ }^{2}$ Kamel Bentabak, ${ }^{3}$ Fatiha Hadjarrab, ${ }^{3}$ Kamel Bouzid. '2, Rue Mahmoud Zani Scala Elbiar; ${ }^{2}$ Cpme Hospital; Surgery; ${ }^{3} \mathrm{Cpmc}$ Hospital; Medical Oncology

\subsection{6/ijgc-2020-ESG0.222}

Introduction/Background To evaluate the infra-millimetric resectability rate using the two approaches, the morbiditymortality rate and the overall survival curves without recurrence.

Methodology From January 2015 to December 2017, 82 patients with ovarian cancer classified stage IIIC by FIGO (International Federation of Gynecologists and Obstetricians), were treated in the oncological surgery department of the CPMC (Center Pierre et Marie Curie), randomized into two groups. First group (G1) including patients who underwent primary debulking surgery and group 2 (G2), patients who underwent primary chemotherapy with platinum salt followed by cytoreductive surgery (interval surgery).

The anatomo-clinical aspect, the histological type, the intraoperative finding, the procedures performed, the results after surgery, the morbidity and mortality and the survival curves were analysed prospectively.

Results The mean age of patients in G1 was 50.05 years (3080 ) and in G2 55.90 years (23-80), the majority of patients were classified ASA I in both groups $(51,2 \%)$, the mean body mass index (BMI) was 29.16 in the G1 and 27.29 in the G2, the most frequent histological type was serous carcinoma in both groups $(69.5 \%)$ of patients. The main procedure performed is a total hysterectomy, bilateral adnexectomy, infra colic or infra gastric omentectomy, pelvic and lumbar aortic dissection and resection of any macroscopically visible lesion.

In some cases, an associated procedure has been performed such as digestive resection, cholecystectomy, peritonectomy, caudal pancreatectomy. Rate of actions performed in G1: 65.8\%; G2: 34.1\%. Rate of R0 obtained (41.4\%) or $51.5 \%$ in G1 and $48.4 \%$ in G2. The operative morbidity was $20.7 \%$ with a rate of $14.6 \%$ in G1 and $6 \%$ in G2.

Conclusion Complete cytoreductive surgery has become a fundamental principle in surgery for peritoneal carcinomatosis. The gold standard for treating advanced ovarian cancer is complete surgery combined with chemotherapy with platinum salt. The sequence of treatment is still debated, but primary surgery seems to be preferred in terms of recurrence-free survival and overall survival when complete resection (R0) is obtained.

Disclosures Pr Chemseddine CHEKMAN: I declare that I have no conflict of interest.
Dr Fatiha GOUAREF: I declare that I have no conflict of interest.

Pr Kamel BENTABAK: I declare that I have no conflict of interest.

Pr Fatiha HADJARRAB: I declare that I have no conflict of interest.

Pr Kamel BOUZID: I declare that I have no conflict of interest.

596

\section{A RANDOMISED PHASE II STUDY OF NINTEDANIB (BIBF1120) COMPARED TO CHEMOTHERAPY IN PATIENTS WITH RECURRENT CLEAR CELL CARCINOMA OF THE OVARY OR ENDOMETRIUM. (NICCC/ENGOT- OV36)}

${ }^{1}$ Rosalind Glasspool, ${ }^{2}$ lain Mcneish, ${ }^{3}$ Anneke Westermann, ${ }^{4}$ Samantha Hinsley, ${ }^{5}$ Jonathan Ledermann, ${ }^{6}$ ssabelle Ray-Coquard, ${ }^{4}$ Claire Lawless, ${ }^{7}$ Nelleke Ottevanger, ${ }^{8}$ Mansoor Raza Mirza, ${ }^{9}$ Jerome Alexandre. ${ }^{1}$ Beatson West of Scotland Cancer Centre; Institute of Cancer Sciences; ${ }^{2}$ Imperial College London; Irdb Building; ${ }^{3}$ Amsterdam University Medical Center; Department of Medical Oncology F4-224; ${ }^{4}$ Cancer Research UK Glasgow Clinical Trials Unit; Institute of Cancer Sciences, University of Glasgow; Beatson West of Scotland Cancer Centre; ${ }^{5}$ Ud Cancer Institute; Cr-UK and Ud Cancer Trials Centre; ${ }^{6}$ Centre Leon Bérard; Hesper Lab Ea 7425; Université Claude Bernard Lyon Est; ${ }^{7}$ Radboud University Medical Centre; Geert Grooteplein Zuid 8 (Route 452); ${ }^{8}$ Copenhagen University Hospital; Rigshospitalet; Department of Oncology 5073; ${ }^{9}$ Université de Paris; Carpem, Cochin-Port Royal; Oncologie Médicale

\subsection{6/ijgc-2020-ESGO.223}

Introduction/Background Clear cell carcinoma (CCC) is a rare subtype of ovarian and endometrial cancer. It carries a poor prognosis and response to chemotherapy in recurrent disease is low. As angiogenesis pathways are activated in CCC, we performed a trial comparing nintedanib (BIBF1120), an orally available, triple kinase inhibitor targeting VEGFR, PDGFR and FGFR with physician's choice of chemotherapy. As the first randomised trial in relapsed CCC, it gives important information on the efficacy and toxicity of both nintedanib and chemotherapy. Here we report the ovarian cancer (OC) results.

Methodology This was an international, multi-centre, randomised, open label phase II, 3 outcome design. Patients were randomised to nintedanib $200 \mathrm{mg}$ PO twice daily or chemotherapy (paclitaxel $(80 \mathrm{mg} / \mathrm{m} 2$ IV Day 1,8,15), pegylated liposomal doxorubicin $(40 \mathrm{mg} / \mathrm{m} 2 \mathrm{IV})$ or topotecan $(4 \mathrm{mg} / \mathrm{m} 2$ IV Day 1,8,15) every 28 days). Treatment was given until disease progression or unacceptable toxicity. The primary endpoint was progression free survival (PFS) in the ovarian cohort. Secondary objectives included overall survival (OS), response rate (RR), disease control rate (DCR) and patient reported outcomes. With 90 OC patients, the study was powered to detect an improvement in median PFS from 3 to 5 months $(\mathrm{HR}=0.6)$ with $>90 \%$ power, $20 \%$ 1-sided significance. A statistically significant PFS difference at the 1-sided $10 \%$ level (Nintedanib superior) would give a clear signal that a phase III study is warranted. A statistically significant result at the 1 -sided $20 \%$ level would require other supportive evidence. EudraCT Ref:2013-002109-73. ISRCTN No: ISRCTN50772895.

Results 91 OC patients were included in the analysis. Median age was 54 years. Median number of previous lines was 2 . After a median follow up of 20.7 months the median PFS was 2.3 months with nintedanib and 1.9 months with chemotherapy (hazard ratio $=0.79,80 \% \mathrm{CI}=(0.58,1.06), \mathrm{p}$ (1-sided) 


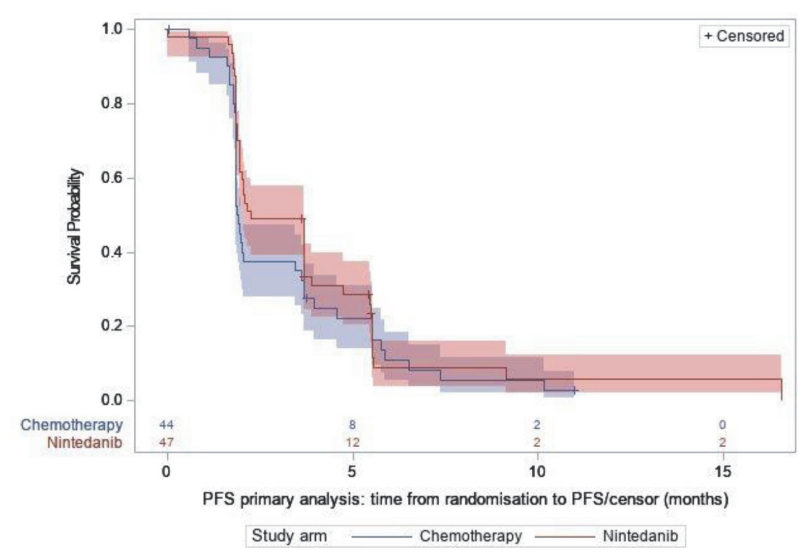

Abstract 596 Figure 1 Progression free survival Kaplan-Meier curves with $80 \%$ Cis

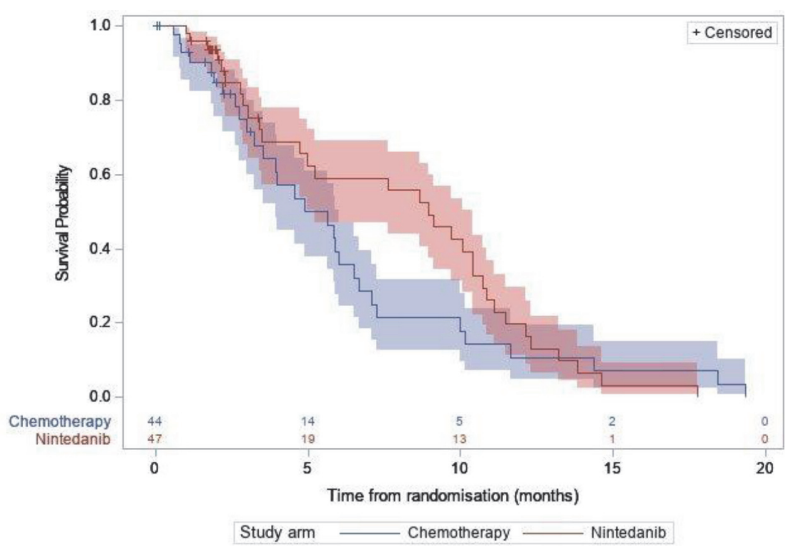

Abstract 596 Figure 2 Overall survival Kaplan-Meier curves, with $80 \% \mathrm{Cls}$

$=0.1521$. Median OS was 9.0 and 4.9 months, respectively. Difference in OS estimates at 6 and 12 months were $19.7 \%$ and $8.9 \%$ demonstrating non-proportional hazards. RR was $2.1 \%$ and $0 \%$, and DCR at 16 weeks was $23.4 \%$ and $9.1 \%$ $($ odds $\quad$ ratio $=5.81, \quad 80 \% \mathrm{CI}=(1.79,18.89), \quad \mathrm{p}(1$-sided $)=0.0276)$ with nintedanib and chemotherapy, respectively.

Conclusion The study failed to demonstrate sufficient activity of nintedanib as a monotherapy to support a phase III trial. However, the benefit in PFS, DCR and OS suggests it may be interesting to combine nintedanib with other agents in OCCC. Chemotherapy is ineffective and the outcomes for women with OCCC are extremely poor confirming the need for continued research into novel targets and therapies. Translational research is on-going (figures 1 and 2).

Disclosures The study was funded by an educational grant from Boehringer Ingelheim and supported by Cancer Research UK Grant ref: C8361/A15600.

Author disclosures Rosalind Glasspool: Grant funding for clinical trials from Boehringer Ingelheim, Lilly/Ignyta and Clovis. Consultancy fees, travel support and/or speaker fees for AstraZeneca, GSK/Tesaro, Clovis, Immunogen and Sotio. Site PI for studies sponsored by AstraZeneca, GSK/Tesaro, Clovis, Immunogen, Lilly and Pfizer.

Samantha Hinsley: none.

Jonathan Ledermann: Advisory Board and Lecture fees from AstraZeneca, Pfizer, Clovis Oncology, MSD/Merck, Eisai,
Artios Pharma, GSK and grant funding from AstraZeneca and MSD/Merck. IDMC fees from Regeneron.

Iain McNeish: Advisory Board fees from AstraZeneca, Clovis Oncology, Tesaro/GSK, Roche, Scancell and Carrick Therapeutics. Institutional grant funding from AstraZeneca.

Jerome Alexandre: Grant funding from Janssen, MSD. Consultancy fees, travel support and/or speaker fees for AstraZeneca, GSK/Tesaro, MSD, Eisai, Novartis, Pharmamar. Site PI for studies sponsored by AstraZeneca, GSK/Tesaro, MSD, Agenus, Merck Serono, Seattle Genetics

Anneke Westermann: none.

Claire Lawless: none

Nelleke Ottevanger: none

Mansoor Raza Mirza: Personal Financial Interests: AstraZeneca, Biocad, Clovis Oncology, Geneos, Genmab, Karyopharm Therapeutics, merck, msd, Oncology Centre, Pfizer, Roche, SeatleGenetics, Sera Prognostics, Sotio, Tesaro-GSK, ZaiLab. Institutional financial interests (study grants): AstraZeneca, Boehringer Ingelheim, Clovis Oncology, Pfizer, Tesaro-GSK

Isabelle Ray-Coquard: Honoraria (self) from Abbvie, Agenus, Advaxis, BMS, PharmaMar, Genmab, Pfizer, AstraZeneca, Roche, GSK, MSD, Deciphera, Mersena, Merck Sereno, Novartis, Amgen, Tesaro and Clovis; honoraria (institution) from GSK, MSD, Roche and BMS; advisory/consulting fees from Abbvie, Agenus, Advaxis, BMS, PharmaMar, Genmab, Pfizer, AstraZeneca, Roche/Genentech, GSK, MSD, Deciphera, Mersena, Merck Sereno, Novartis, Amgen, Tesaro and Clovis; research grant/funding (self) from MSD, Roche and BMS; research grant/funding (institution) from MSD, Roche, BMS, Novartis, AstraZeneca and Merck Sereno; and travel support from Roche, AstraZeneca and GSK.

\section{PERITONEAL CARCINOMATOSIS INDEX AS A PREDICTOR OF CYTOREDUCTIVE OUTCOMES IN PATIENTS WITH ADVANCED OVARIAN CANCER}

${ }^{1}$ Maria Yakovleva, ${ }^{1}$ Olga Smirnova, ${ }^{2}$ Elena Ulrikh, ${ }^{1}$ Elvina Bakaeva, ${ }^{1}$ Anna Petrova, ${ }^{1}$ Ksenia Kozyreva, ${ }^{1}$ Lev Shevkunov, ${ }^{1}$ Nikolay Mikaya, ${ }^{1}$ Adel Urmancheeva, ${ }^{1}$ Igor Berlev. ${ }^{1} N$. N. Petrov National Medical Research Center of Oncology; ${ }^{2}$ Almazov National Medical Research Centre; North-Western State Medical University, N.N.Petrov National Medical Research Centre of Oncology; Oncology

\subsection{6/ijgc-2020-ESG0.224}

Introduction/Background In our study, we evaluated the peritoneal carcinomatosis index (PCI) and to study the influence of PCI on the cytoreductive surgery outcomes in patients with advanced ovarian cancer.

Methodology We analyzed the results of 133 patients with serous high-grade ovarian cancer FIGO IIIC-IVB stages. PCI was evaluated at 13 regions (score $0-3$ for each region) via pelvic MRI, chest and abdominal CT and staging laparoscopy (S-LPS.)

Results Were performed 23\% (31/133) of primary and $77 \%$ $(102 / 133)$ of interval debulking surgery (DS), 68\% (90/133) of them were optimal (no residual disease, ODS) and 32\% (43/ 133) were suboptimal (residual tumor of $>1 \mathrm{~cm}$, SDS). The frequency of ODS for PCI $0-15$ score was $60-86 \%$, for PCI 15-20 score - 9\%, PCI> 20 score - 0\%. Preoperative and intraoperative determination of the PCI was equal in $58 \%$. In $19.8 \%$ PCI was overestimated during preoperative examination, while the use of laparoscopy evaluated PCI allowed to perform an ODS in these cases. In 21\% PCI was 